Dhaka Univ. J. Biol. Sci. 29(2): 191-199, 2020 (July)

\title{
ANALYZING THE EFFECT OF FOREIGN LANGUAGE ON DECISION MAKING : A STUDY FROM BANGLA LANGUAGE PERSPECTIVE
}

\author{
A.M. Zahidul Hague, Naima Nigar, Arifa Rahman, \\ FARIEA BAKUL* AND SYFIN RUBYAT ${ }^{1}$ \\ Department of Psychology, University of Dhaka, Dhaka-1000, Bangladesh
}

Key words: Foreign language, Decision biases, Bangla language, Perspective

\begin{abstract}
Decision making is one of the crucial aspects of our daily life. In terms of making any decision language plays a vital role either directly or indirectly. The purpose of the present study was to investigate the impact of Bangla language (native) and foreign language (English) on decision making. It was hypothesized that foreign language would show fewer decision biases. Total 80 participants (40 males, 40 females) took part in this experiment. Results from Chi-square test revealed that while making decisions in Bangla language under loss-frame $68.7 \%$ participants preferred to choose risk option over sure option. On the contrary in gain-frame only $29.9 \%$ participants preferred the risk option $\left(\chi^{2}(1, \mathrm{~N}=80)=24.059\right.$, $\mathrm{p}<0.001)$. This decision biasness was significantly minimized in English language. In loss-frame $56.1 \%$ participants preferred the risk option over sure option and in gain-frame $43.6 \%$ participants preferred the risk option, $\chi^{2}(1, \mathrm{~N}=80)=$ $2.502, \mathrm{p}>0.05$.
\end{abstract}

\section{Introduction}

The capacity to make the decisions based on logic and reasoning makes us unique from other animals. Everyday people make different decisions starting from home to the office. In general, decision-making generally is regarded as a problem-solving activity which is considered as giving most fruitful or a satisfactory result at least. Decision making is the process which can be narrated as more or less rationale, some cases irrational ${ }^{(1)}$. The process of making decision depends on various factors, such as, the type of problem ${ }^{(2)}$, the individuals stress level(3), the mood of the participant ${ }^{(4)}$ and the language in which the problem is presented ${ }^{(5)}$.

Here, it was investigated how human decision making process gets influenced through framing effect and language. The framing effect means a person having varying opinion about similar problems because of different presentation style(6). Gain and loss are two ways of describing the same consequence. People pick for sure choice when a situation is presented in "gain" manner but choose to take a risk when the same situation is presented in "loss" manner. According to prospect theory ${ }^{(7)}$, people consider loss more significant than an equal gain. In an experiment where the participants were presented

*Author for correspondence: < fariea.bakul@du.ac.bd>. ${ }^{1}$ Department of Linguistics, University of Dhaka, Dhaka-1000, Bangladesh. 
with a problem and were given two options from which they could pick only one. The result revealed that $72 \%$ of the participants have chosen the sure option when the options were presented in a gain/positive form, but only $22 \%$ of them choose the sure option in the scenario where the options were presented in loss/negative form ${ }^{(6)}$. Thus, it can be said that the framing effect is one of the important factors in influencing our decision.

Bilingualism not only refers to an individual's linguistic performance, but it also affects cognitive processes as well( ${ }^{(8)}$. Studies exploring the relationship between language control and cognitive control revealed that bilingualism enhances executive control ${ }^{(8)}$. Executive control, an aspect of human cognition, usually refers to the individual's control over his/her thoughts and actions ${ }^{(9)}$. Executive control can also be defined as "those types of actions individuals perform to ourselves and direct at ourselves so as to accomplish selfcontrol, goal-directed behaviour, and the maximization of future outcomes"(10). Thus, decision making, which is a mental activity can be affected by the languages in which participants are controlled to code-switch to respond on their decisions according to the language in which problem statements are presented.

Philosophers from the early era of philosophy used to believe that different languages influence thinking in different ways. Linguists hypothetically believed that speakers of different languages think differently since the system, such as the structure of any language can affects the 'world view' or the cognition of a speaker(11). This claim is supported by 'Linguistic Relativity and Determinism Hypothesis' which is also known as the Sapir-Whorf Hypothesis or Whorfian Hypothesis. Linguistic Relativity, the first notion of this hypothesis asserts that languages are relative and thus they vary in their expressions of concepts since the speakers of a particular language are born and raised in a particular context of culture(6). Linguistic determinism, the second notion of this hypothesis claims that the structure of a particular language determines the way of thinking of the speakers of that language. Thus, it can be argued that the structure of a language influences the way of thinking of the speaker of that language, the capacity of using a different language of a speaker can affect the executive control of that speaker ${ }^{(11)}$. In a study it was demonstrated that individual differences in language abilities were also manifested by differences in brain functioning(12). To control this individual difference, it is best to use a repeated measurement design to see the framing effect of language on decision making.

In original study two different groups of participants were used to observe the framing effect of language on decision making. It was proved from different researches that there is an individual difference in comprehending Language. To control this individual difference, it is best to use repeated measurement design to see the framing effect of language on decision making. The repeated measurement design would not only reduce individual differences on language comprehension ability but it would also reduce the individual differences of foreign language capacity. Thus, in the present research we just redesigned the previous research and used Repeated Measurement Design instead of 
Between Group Design. In addition, this kind of research would be the first in Bangla language.

The purpose of the study was to investigate the impact of native language (Bangla) and foreign language (English) on the decision making. It was hypothesized that foreign language would show a fewer decision biases.

\section{Materials and Methods}

In this experiment, a total number of 80 students (40 males and 40 females) of Dhaka University, Bangladesh took part as participants. They were selected in a purposive manner. The criterion for including the participants in the study was their proficiency in Bangla as well as in English language. English would be their second language and they must have learned English since their primary school. To ensure about their proficiency in English language, university students who were taking their undergraduate degree in English were recruited as participants. Their age ranged from 24 - 26 years.

In this experiment repeated measurement ABBA design was used. The repeated measurement design would not only reduce individual differences in language comprehension ability but it would also reduce the individual differences of foreign language capacity.

The participants were presented with a sheet consisting of four problems. Among them, two were in Bangla and two in English and in each language one of the options was in the gain frame and another one was in loss frame. Prior to every problem, there was a short story regarding an incident which was related to that particular problem, and then the problem was stated with two possible solutions. The number of sentences used in describing each incident was equal. Each of the problems dealt with life-threatening situations and the gain-loss ratio was equal in each of them.

Participants were selected in a convenient manner. Among them who were only bilingual and had good command in their second language (English) were recruited for this study. At first, they were briefed about the purpose of the study and also assured that their responses and personal information would be kept confidential and only be used for research purposes. After getting their verbal consent, the experiment started. Participants were given the instruction both verbally and in written format. Then they were provided with the problem sheets attached to the appendix. The first one was 'Bangla-gain' frame, the second one was 'English-loss frame', the third one was 'English gain' frame and the fourth one was 'Bangla-loss' frame. Every problem sheet consisted of two options, one was 'sure' option and another one was 'risk' option. The problem responses were also counter balanced. The problems and solutions were carefully constricted as it should be really easy to understand. The ratio of loss-gain was kept equal in all the problems.

The presentation order of solutions in every problem was interchanged to diminish the biasness toward a certain option. The participants were told to choose a solution in 
every problem sheet (attached to the appendix). After collecting all the data, the participants were thanked and wished to have a good day.

\section{Results and Discussion}

For investigating the effect of foreign language on decision making 2 (Language) $\times 2$ (Frame) $\chi^{2}$ test was applied. Before conducting the main test, for eliminating any possible gender difference $\chi^{2}$ test was performed for each frame in their corresponding language.

It can be observed from the Fig. $1 \mathrm{~A}$ and $\mathrm{B}$ that in case of the native language (Bangla) the percentage of male and female participants in choosing risk and sure option both for loss and gain frame is almost close (e.g., 67.5 male choose risk option compared to 75.0 female choose in loss frame). However, the test of Pearson Chi-square indicated this difference as non-significant (Loss frame: $\chi^{2}=0.549, p=0.459$ and gain frame: $\chi^{2}=2.051$, $\mathrm{p}=0.152)$.
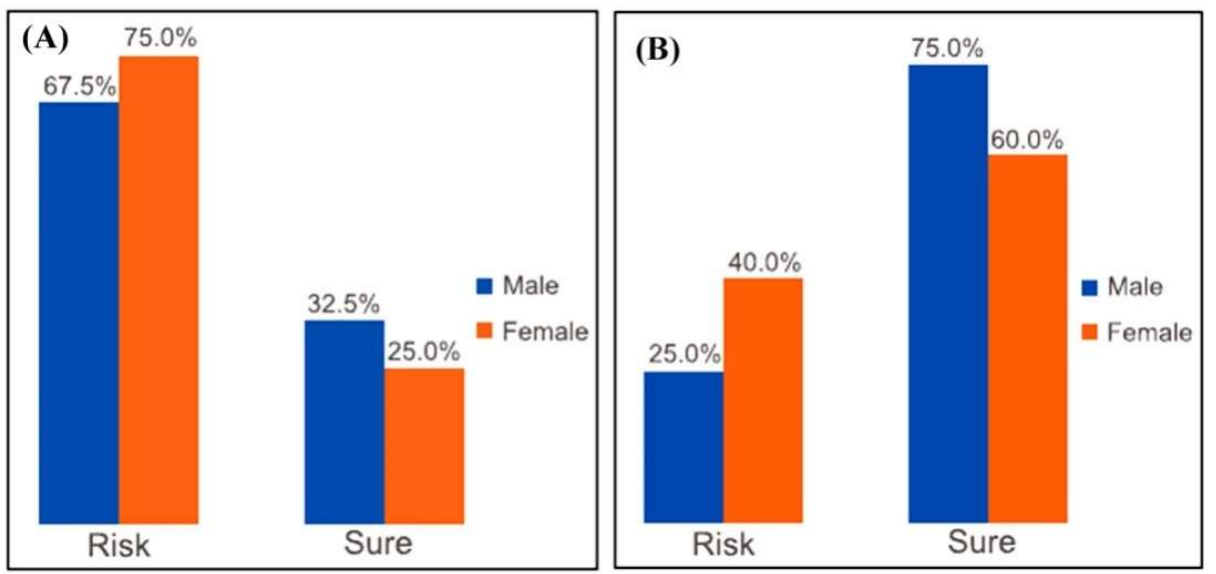

Fig. 1. Representing gender differences in percentage for Bangla loss frame (A) and Bangla gain frame (B).

Similar findings were observed for foreign language (English) loss and gain frame (Fig. 2). The value of the Pearson Chi-square indicated no significant gender differences for the English loss frame $\left(\chi^{2}=0.818, p=0.366\right)$ and English gain frame $\left(\chi^{2}=0.808, p=0.369\right)$.

Finally, to investigate the framing effect of the language on decision making $\chi^{2}$ test was performed. As shown in Table 1, the framing effect for the native language (Bangla) was found to be significant $\left(\chi^{2}(1, N=80)=24.059, p<0.001\right)$. Among the 80 participants, in lossframe problem, $35.6 \%$ of them showed preference for the risk option, whereas only $14.4 \%$ of the participants in loss-frame problem showed preference in the sure option and in gainframe problem. Only $16.3 \%$ of them preferred risk option, whereas only $33.8 \%$ of the 
participants in gain-frame problem showed preference the sure option. Thus, it can clearly be stated that the native language influences our decision making.
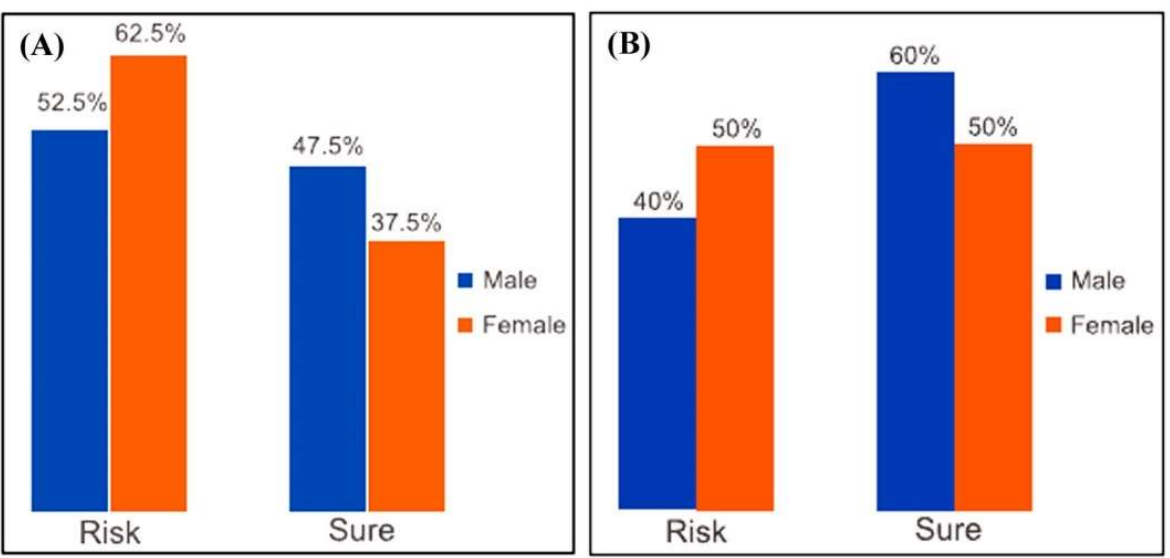

Fig. 2. Representing gender differences for English loss frame (A) and English gain frame (B).

Table 1. Cross tabulation of loss and gain frame $\times$ risk and sure option for native language.

\begin{tabular}{|c|c|c|c|c|c|}
\hline \multirow{2}{*}{$\begin{array}{l}\text { Choice of } \\
\text { option }\end{array}$} & \multicolumn{2}{|c|}{ Frame } & \multirow[t]{2}{*}{$\chi^{2}$} & \multirow[t]{2}{*}{ Df } & \multirow[t]{2}{*}{$p$} \\
\hline & Loss & Gain & & & \\
\hline \multirow[t]{2}{*}{ Risk } & 57 & 26 & 24.059 & 1 & $<0.001$ \\
\hline & $68.7 \%$ & $31.3 \%$ & & & \\
\hline \multirow[t]{2}{*}{ Sure } & 23 & 54 & & & \\
\hline & $29.9 \%$ & $70.1 \%$ & & & \\
\hline
\end{tabular}

Table 2 shows the framing effect on foreign language (English). Among the total 80 participants, in loss-frame problem, $28.8 \%$ of them showed preference for the risk option, whereas only $21.3 \%$ of the participants in loss-frame problem showed preference in the sure option, in gain-frame problem $22.5 \%$ of them preferred the risk option, whereas only $27.5 \%$ of the participants in gain-frame problem preferred the sure option. However, these differences were non-significant $\chi^{2}(1, \mathrm{~N}=80)=2.502, \mathrm{p}>0.05$.

As the present study investigated whether the foreign language (English) reduces decision biasness or not results revealed that there was no significant difference between gender which was supportive of previous research findings ${ }^{(13)}$. In another study it was found that females were less prone to risk-taking than males. They suggested that males and females adopted different strategies while making a business decision ${ }^{(14)}$. One important fact was that there was no prior research which focused on decision biasness between genders in life-threatening situations. Future studies should emphasis on this issue with larger samples of different ages. 
Table 2. Cross tabulation of loss and gain frame $\times$ risk and sure option for foreign language.

\begin{tabular}{llllll}
\hline \multirow{2}{*}{$\begin{array}{l}\text { Choice of } \\
\text { option }\end{array}$} & \multicolumn{2}{c}{ Frame } & \multirow{2}{*}{$\chi^{2}$} & df & $\mathrm{p}$ \\
\cline { 2 - 3 } & Loss & Gain & & & \\
\hline Risk & 46 & 36 & 2.502 & 1 & 0.114 \\
& $56.1 \%$ & $43.9 \%$ & & & \\
Sure & 34 & 44 & & & \\
& $43.6 \%$ & $56.4 \%$ & & & \\
\hline
\end{tabular}

Another finding of the present study was that there were significant differences between languages. Table 1 shows, the framing effect in the native language, Bangla. Among the total 80 participants, in loss-frame, $68.7 \%$ preferred the risk option. On the other hand, in the gain-frame problem, $29.9 \%$ preferred the risk option. $\chi^{2(1, N=80)}=24.059$, $\mathrm{p}=<0.001$. Also there is a significant difference in the preferred option for the native language.

But in the case of the foreign language Table 2 shows the framing effect in the foreign language, English. Among the total 80 participants, in loss-frame problem $56.1 \%$ preferred the risk option and in gain-frame problem $43.6 \%$ preferred the risk option $\quad \chi^{2(1, \mathrm{~N}=80)}=$ $2.502, p>0.05$. According to these results, there is no significant difference in decision making in a foreign language. This is exactly what the present experiment hypothesized and findings of the current experiment support our hypothesis. The foreign language reduces decision biasness.

The result of the current experiment suggests that pupils usually rely more on systematic process while making decisions for any topic given or presented in a foreign language. One prominent reason behind this is the emotional resonance ${ }^{(5)}$. It is associated with the use of a foreign language. There is also evidence that amygdala plays a key role in framing effect which suggests that it results from a strong emotional attraction to sure gains and a strong aversion to sure losses ${ }^{(15)}$. Generally, it may not make any sense that people would opt for different solutions for the same problem depending on the languages it is presented. But the nature of the language does have a systematic effect.

In different working sectors such as business deal, developing new policies, participating in political polls etc. people always have to make decisions. Since foreign language plays an important role in decision making so it is important to investigate how this affects our decision-making process. The present finding would really help us a lot to understand business psychology. Consumers decisions are heavily manipulated by language and its framing. Nowadays workers are keen to conduct online research, the findings of our research can help them a lot. It would be important to know when participants used a foreign language in order to interpret results accurately. More generally, keeping in mind that now in our daily life people are using foreign language 
often enough. Our findings can be implemented from important business decisions to daily life decisions.

\section{References}

1. Brockmann EN and WP Anthony 2002. Tacit knowledge and strategic decision making. Group \& Organization Management 27(4): 436-455. doi:https://doi.org/10.1177/1059601102238356

2. Bell RL and NG Lederman 2003. Understandings of the nature of science and decision making on science and technology-based issues. Science Education 87(3): 352-377. doi.org/10. 1002/sce.10063

3. Kowalski-Trakofler KM, C Vaught and T Scharf 2003. Judgment and decision making under stress: An overview for emergency managers. International Journal of Emergency Management 1(3): 278-289. doi.org/10.1504/IJEM.2003.003297

4. Yuen KS and TM Lee 2003. Could mood state affect risk-taking decisions? Journal of Affective Disorders 75(1): 11-18.

5. KeysarB, SL Hayakawa and SG AN 2012. The foreign-language effect: Thinking in a foreign tongue reduces decision biases. Psychological Science 23(6): 661-668. doi: 10.1177/ 0956797611432178 .

6. Tversky Aand D Kahneman 1981. The framing of decisions and the psychology of choice. Science 211(4481): 453-458. doi: 10.1126/science.7455683

7. Kahneman D and A Tversky 1979. Prospect theory: An analysisof decision under risk. Econometrica 47: 263-291. doi:10.2307/1914185

8. Woumans E 2015. Effects of Bilingualism on Cognition. Gent: Universiteit Gent.

9. Brocki KC 2007. Executive Control Processes:Dimensions, Development and ADHD. Uppsala: Upsala Universitet.

10. Barkley RA 1997.ADHD and the Nature of Self Control. New York: Gulford.

11. Slobin DI 1979. Psycholinguistics Second Edition. Glenview, Illinois: Scott, Foresman and Company.

12. Prat CS 2011. The brain basis of individual differences in language comprehension abilities. Linguistics and Language Compass 5(9): 635-649. https://doi.org/10.1111/j.1749-818X. 2011.00303.x

13. Schubert R, M Brown, M Gysler and HW Brachinger 1999. Financial decision-making: Are women really more risk-averse? American Economic Review 89(2): 381-385.doi: 10.1257/aer.89.2.381

14. Powell M and D Ansic 1997. Gender differences in risk behaviour in financial decision-making: An experimental analysis. Journal of Economic Psychology 18(6): 605-628. doi.org/10. 1016/S0167-4870(97)00026-3

15. Kahneman D and S Frederick 2007. Frames and brains: Elicitation and control of response tendencies. Trends in Cognitive Sciences, 11(2): 45-46. doi.org/10.1016/j.tics.2006.11.007. 


\section{Appendix-1}

1. Problem sheet used in the experiment.

একটি ব্রিজের উপর রেল লাইনের একই ট্র্যাকে মুখোমুখি হয়ে ২ টা টেন আসছে। টেনে নোট ২০,০০০ যার্রী আছে। তাদের মধ্যে ৬,৬৬৭ জন মানুষ সাঁতার পারে। টেনের ড্রাইভার বুঝতে পেল সামনে ঘটতে যাচ্ছে ভয়ানক বিপদ ততক্ষণে পাণের ট্র্যাকে ফিরে যাওয়ার মতো সময় আর নেই। এখন দুটি উপায় আছে, ব্রিজ থেকে পাশের নদীতে টেনটিকে নামিয়ে দেয়া অথবা হার্ড ব্রেক করা এবং আশা করা যে সাল্নের টেনটিও হার্ড র্রেক করে থামাতে সক্ষম হরে। এই মুহূর্ত্ত ড্রাইভার যদি কিছু না করে তাহলে ২০০০০ জন মানুষই মারা যাবেন ।

উপায়-১ঃ অবলম্বন করে ব্রিজের পাশের নদীত টেনটি নামিয়ে দিলে ঠিক ৬,৬৬৭ জন মানুষ নিশ্চিত বেচে যাবেন ।

উপায়-২ঃ অবলম্বন করে হার্ড ব্রেক করলে ৩৩.৪\% সম্ভাবনা আছে ২০০০০ জন যাত্রী বেটে যাবেন এবং ৬৬.৬\% সম্ভাবনা আছে কেউ বাচবেন না।

আপনি হলে কোনটি বেছে নিতেন?

উত্তর ঃ

একটি হোটেলে হঠাৎ জংগী হামলা হল। জংগীরা এখন ৩০,০০০ জন কে জিম্মি করল এবং মুক্তিপণ চাইলো প্রচুর পরিমাণ টাকা। সার্বিক অবস্থা পর্যবেক্ষণের পর কমান্ডো বাহিনী বুঝতে পারলো তাদের হাতে দুটি উপায় আছে। মুক্তিপণের টাকা দিয়ে দিতে পারে অথবা তারা জংগীদের উপর হামলা করতে পারে। কোন কিছু না করলে ৩০,০০০ জন মানুষই মারা যাবেন।

উপায়-১ঃ অবলম্বন করে তারা মুক্তিপণের টাকা দিয়ে দিলে ৩৩.৪\% সম্ভাবনা আছে কেউই মারা যাবেন না এবং ৬৬.৬\% সম্ভাবনা আছে ৩০,০০০ জনই মারা যাবেন।

উপায়-২ঃ অবলম্বন করে তারা জংগীদের উপর হামলা করলে ক্রস্ফায়ারে ঠিক ২০,০০০ জন জিম্মি মানুষ নিশ্চিত মারা যাবেন।

আপনি হলে কোন উপায় টি বেছে নিতেন?

উত্তরঃ

Recently a deadly virus disease has been emerged. At the moment, it has turned into an epidemic form in the few countries of West Africa. People are dying rapidly. A matter of relief is that recently scientists have come up with 2 types of medicines for this disease but they can produce only one type of medicine. Currently a total 60,000 people are infected by this virus. Without any medicine 60,000 people will die.

1. if you choose medicine A - precisely 40,000 people will die. 
2. if you choose medicine B - there is $33.3 \%$ chance that no one will die and $66.7 \%$ chance that all 60,000 people will die.

Which medicine would you choose?

Ans :

An orphanage was on fire and Fire brigade rushed to the spot. There were two entrance gates of the building - Gate A and Gate B. They could enter only through one. They had the information that 13,333 children are stuck somewhere near entrance 'A'. Total 40,000 children is stuck in the building. If they don't take any initiative 40,000 children will die.

1. if they choose entrance ' $\mathrm{B}$ ' - there is $33.3 \%$ chance that all 40,000 children will be saved and $66.7 \%$ chance that no one will be saved.

2. if they choose entrance 'A' - precisely 13,333 children will be saved.

Which entrance would you choose?

Ans :

প্রিয় পরীক্ষক, এখানে একটি স্কেল দেয়া আছে। স্কেলটি আপনার ইংরাজী ভাষার দক্ষতা প্রকাশ করবে। আপনার নিজস্ব ধারণা অনুযায়ী আপনি ইংরেজী তে কেমন সেটি ১ - ১০ এর মধ্যে প্রকাশ করবেন। এখানে ১ সর্বণিয্ন এবং ১০ সর্বোচ্চ। আপনার মতামত নিজ নিজ ঘরে টিক চিহ্ দিয়ে প্রকাশ করুন।

Self rating scale

\begin{tabular}{|l|l|l|l|l|l|l|l|l|l|l|}
\hline Abilities & 1 & 2 & 3 & 4 & 5 & 6 & 7 & 8 & 9 & 10 \\
\hline Reading & & & & & & & & & & \\
\hline Writing & & & & & & & & & & \\
\hline Speaking & & & & & & & & & & \\
\hline Understanding & & & & & & & & & & \\
\hline
\end{tabular}

